A Block-Diagonal Algebraic Multigrid Preconditioner for the Brinkman Problem

P. S. Vassilevski, U. Villa

January 12, 2012

12th Copper Mountain Conference on Iterative Methods, 2012 Copper Mountain Resort, CO, United States March 25, 2012 through March 30, 2012 
This document was prepared as an account of work sponsored by an agency of the United States government. Neither the United States government nor Lawrence Livermore National Security, LLC, nor any of their employees makes any warranty, expressed or implied, or assumes any legal liability or responsibility for the accuracy, completeness, or usefulness of any information, apparatus, product, or process disclosed, or represents that its use would not infringe privately owned rights. Reference herein to any specific commercial product, process, or service by trade name, trademark, manufacturer, or otherwise does not necessarily constitute or imply its endorsement, recommendation, or favoring by the United States government or Lawrence Livermore National Security, LLC. The views and opinions of authors expressed herein do not necessarily state or reflect those of the United States government or Lawrence Livermore National Security, LLC, and shall not be used for advertising or product endorsement purposes. 


\title{
A BLOCK-DIAGONAL ALGEBRAIC MULTIGRID PRECONDITIONER FOR THE BRINKMAN PROBLEM
}

\author{
PANAYOT S. VASSILEVSKI AND UMBERTO VILLA
}

\begin{abstract}
The Brinkman model is a unified law governing the flow of a viscous fluid in cavity (Stokes equations) and in porous media (Darcy equations). In this work, we explore a novel mixed formulation of the Brinkman problem. Introducing the flow's vorticity as additional unknown, this formulation leads to a uniformly stable and conforming discretization by standard finite element (Nédélec, Raviart-Thomas, piecewise discontinuous). Based on stability analysis of the problem in the $H($ curl $)-H($ div $)-L^{2}$ norms, we derive a scalable block diagonal preconditioner which is optimal in the constant coefficient case. Such preconditioner is based on the auxiliary space AMG solvers for $H$ (curl) and $H$ (div) problems available in hypre $([10])$. The theoretical results are illustrated by numerical experiments.
\end{abstract}

\section{INTRODUCTION}

The Brinkman equations describe the flow of a viscous fluid in cavity and porous media. It was initially proposed in [1], [2] as a homogenization technique for the Navier-Stokes equations. Typical applications of this model are in underground water hydrology, petroleum industry, automotive industry, biomedical engineering, and heat pipes modeling.

Mathematically speaking the Brinkman model is a parameter-dependent combination of the Darcy and Stokes models. Since in real applications the number and the locations of the Stokes-Darcy interfaces might not be known a priori, the unified equations in the Brinkman model represent an advantage over the domain decomposition methods coupling the Darcy and the Stokes equations. However, the high variability in the PDE coefficients, that may take extremely large or small values, negatively affects the conditioning of the discrete problem which poses a substantial challenge for developing efficient preconditioners for this problem.

Another challenging aspect of the Brinkman model is the construction of a stable finite element discretization ([21]). In [14], it is proved that inf-sup compatible finite element for Stokes (Taylor Hood, P2-P0, Crouzeix-Raviart - P0, mini elements) will lead to non-convergent discretizations in the limit Darcy case (viscosity $\nu \rightarrow 0$ or inverse permeability $k \rightarrow \infty$ ) and that Raviart-Thomas elements for the discretization of the velocity field fail in the case $\nu \neq 0$, since they are not $H^{1}$-conforming. Numerous different approaches have been proposed in the literature to address the numerical stability of the discretization. Among those, in [7] and [8], the authors introduce jumps penalization on the normal component of the velocity field or on the pressure field to stabilize the Crouzeix-Raviart and P0 finite elements or P1-P0 finite elements, respectively. In [9], an augmented Lagrangian approach and a least squares stabilization is explored in order to use inf-sup compatible Taylor-Hood elements also in the Darcy case, while in [14] high order non-conforming elements are investigated.

In the present paper, we consider the mixed formulation of the Brinkman problem proposed by the authors in [20]. Following what has already been done for the Stokes problem ([6], [3]), the authors introduced the (scaled) vorticity as additional unknown. The well-posedness analysis of the mixed

Key words and phrases. Brinkman problem; Stokes-Darcy coupling; saddle point problems; block preconditioners; algebraic multigrid.

This work was performed under the auspices of the U.S. Department of Energy by Lawrence Livermore National Laboratory under Contract DE-AC52-07NA27344. 
formulation was based on the Hilbert complex structure for the Hodge Laplacian, and the numerical stability of the method was guaranteed by an analogous results on the discrete level. The particular choice of Nédélec, Raviart-Thomas and piecewise discontinuous elements, in fact, reproduces the same embedding and mapping properties of the continuous spaces in the finite elements spaces. The linear system obtained after finite element discretization has a symmetric saddle point form. In contrast to the penalization methods for the Brinkman problem, our approach provides a conforming discretization by standard finite elements. Discretization errors in the $H$ (div)-norm of the velocity and in the $L^{2}$ norm of the pressure show uniform decay rates with respect to the inverse permeability coefficient $k(x)$. Only the (scaled) vorticity is approximated with less accuracy as we approach the Darcy limit.

A main disadvantage of the mixed formulation approach is that the Hodge decomposition holds only for particular sets of boundary conditions ([3]).

In this work, we focus on the development of effective preconditioning techniques for the discrete saddle point problem. Following the approach in [15], we construct a block diagonal preconditioner with optimal convergence properties based on the stability analysis of the continuous problem. Such preconditioner has on the main diagonal the finite element matrices corresponding to the $H$ (curl), $H$ (div), and $L^{2}$ norms involved in the stability estimates. To improve the efficiency of the preconditioner, we resort to the recently developed auxiliary space multigrid preconditioner for $H(\operatorname{div})$ and $H$ (curl) problems.

In Section 1, we briefly derive the mixed formulation of the Brinkman problem based on the Hodge Laplacian, and we provide a stability estimate. In Sect. 2, we address the numerical discretization of the mixed formulation with Nédélec, Raviart-Thomas and piecewise polynomial discontinuous finite element which leads to a large sparse saddle point linear system. In Sect. 3, we derive an optimal preconditioner with respect to the mesh size. We also investigate an augmented Lagrangian approach in order to improve the robustness of the preconditioner with respect to the PDE coefficients. Finally, in Sect. 4 we present numerical results for the case of constant coefficient and smoothly varying coefficient.

\section{Mixed formulation of the Brinkman Problem}

Let $\Omega$ be a bounded domain in $\mathbb{R}^{d}$ with a regular enough boundary $\partial \Omega$ that has well-defined unit outward normal vector $\mathbf{n} \in \mathbb{R}^{d}$. The generalized Brinkman problem reads

$$
\begin{cases}-\nu \Delta \mathbf{u}+k(\mathbf{x}) \mathbf{u}+\nabla p=\mathbf{f}(\mathbf{x}) & \forall \mathbf{x} \in \Omega \\ \operatorname{div} \mathbf{u}=g(\mathbf{x}) & \forall \mathbf{x} \in \Omega \\ \mathbf{u} \times \mathbf{n}=\mathbf{g} & \text { on } \partial \Omega \\ -p+\nu \operatorname{div}(\mathbf{u})=h & \text { on } \partial \Omega\end{cases}
$$

where $\nu \geq 0$ is the fluid viscosity and $k(x)$ is the inverse permeability of the medium. The challenge of this problems is when the coefficient $k=k(\mathbf{x})$ takes two extreme values $\mathcal{O}(1)$ and $\mathcal{O}(1 / \epsilon)$ in different parts of $\Omega$. In the part of the domain with $k=\mathcal{O}(1)$, the PDE behaves like a Stokes problem, whereas in the rest of the domain, it behaves like Darcy equation.

In the present work, for simplicity, we assume natural boundary conditions on $\partial \Omega$. However, other set of boundary conditions, like the essential boundary conditions $\left(\mathbf{u} \cdot \mathbf{n}=u_{n}, \boldsymbol{\sigma} \times \mathbf{n}=\boldsymbol{\sigma}_{\tau}\right)$, can also be treated in a similar way. For the Hodge Laplacian, natural boundary conditions are also known in the literature as electric boundary conditions while the essential ones as magnetic boundary conditions due to the close relation with Maxwell's equations. In our work, we do not consider the case of full Dirichlet boundary condition, as the mixed formulation is harder to analyze; it leads to suboptimal discretization error behavior ([3]).

To obtain a mixed formulation of the Brinkman problem (1.1) we exploit the vector calculus identity

$$
\Delta \mathbf{u}=\nabla \operatorname{div} \mathbf{u}-\operatorname{curl} \operatorname{curl} \mathbf{u},
$$


and we define the (scaled) vorticity variable

$$
\boldsymbol{\sigma}=\varepsilon \operatorname{curl}(\mathbf{u}), \varepsilon=\sqrt{\nu} .
$$

After some straightforward manipulations, the mixed formulation reads

$$
\begin{cases}\boldsymbol{\sigma}-\varepsilon \operatorname{curl}(\mathbf{u})=0, & \mathbf{x} \in \Omega \\ \varepsilon \operatorname{curl}(\boldsymbol{\sigma})-\varepsilon^{2} \nabla(\operatorname{div} \mathbf{u})+k(x) \mathbf{u}+\nabla p=\mathbf{f}(x), & \mathbf{x} \in \Omega \\ \operatorname{div}(\mathbf{u})=g(\mathbf{x}), & \mathbf{x} \in \Omega \\ \mathbf{u} \times \mathbf{n}=\mathbf{g}, & \text { on } \partial \Omega \\ -p+\varepsilon^{2} \operatorname{div}(\mathbf{u})=h, & \text { on } \partial \Omega .\end{cases}
$$

1.1. Functional spaces and orthogonal decompositions. We now introduce the notation used throughout the paper. For vectorial functions $\mathbf{u}, \mathbf{v} \in\left[L^{2}(\Omega)\right]^{d}$ and scalar functions $p, q \in L^{2}(\Omega)$, we let $(\mathbf{u}, \mathbf{v})=\int_{\Omega} \mathbf{u} \cdot \mathbf{v} d \Omega$ and $(p, q)=\int_{\Omega} p q d \Omega$. Similarly, we denote by $\|\mathbf{v}\|$ and $\|p\|$ the norms induced by the respective inner products.

To come up with the weak formulation of the system (1.2), we introduce the functional spaces $\mathbf{Q}, \mathbf{R}$ and $W$, defined as

- $\mathbf{Q} \equiv H(\operatorname{curl}, \Omega):=\left\{\boldsymbol{\sigma} \in L^{2}(\Omega) \mid \operatorname{curl} \boldsymbol{\sigma} \in L^{2}(\Omega)\right\}$, equipped with the norm

$$
\|\boldsymbol{\tau}\|_{\mathbf{Q}}^{2}=\|\boldsymbol{\tau}\|^{2}+\|\operatorname{curl}(\boldsymbol{\tau})\|^{2}
$$

- $\mathbf{R} \equiv H(\operatorname{div}, \Omega):=\left\{\mathbf{u} \in L^{2}(\Omega) \mid \operatorname{div} \mathbf{u} \in L^{2}(\Omega)\right\}$, equipped with the norm

$$
\|\mathbf{v}\|_{\mathbf{R}}^{2}=\|\mathbf{v}\|^{2}+\|\operatorname{div}(\mathbf{v})\|^{2} .
$$

- $W \equiv L^{2}(\Omega)$, equipped with the norm

$$
\|q\|_{Q}^{2}=\|q\|^{2}
$$

We denote with $\mathbf{Q}^{*}, \mathbf{R}^{*}$, and $W^{*}$ the dual spaces of $\mathbf{Q}, \mathbf{R}$, and $W$, respectively.

Then we recall the orthogonal decomposition in $L^{2}, H$ (curl) and $H($ div). Such decompositions are of utmost importance in the stability analysis of the mixed problem ([20]), and for the derivation of the auxiliary space multigrid preconditioners for such spaces ([4], [5]). Letting $\mathbf{X}=\{\boldsymbol{\tau} \in H(\operatorname{curl}) \mid \exists \psi \in$ $\left.H^{1}: \boldsymbol{\tau}=\nabla \psi\right\}$ and $\mathbf{Y}=\{\mathbf{v} \in H(\operatorname{div}) \mid \exists \boldsymbol{\psi} \in H(\operatorname{curl}): \mathbf{v}=$ curl $\boldsymbol{\psi}\}$, we have the following orthogonal decompositions:

$$
L^{2}=\mathbf{X} \oplus \mathbf{Y}, \quad H(\operatorname{curl})=\mathbf{X} \oplus \mathbf{X}^{\perp}, \quad H(\operatorname{div})=\mathbf{Y} \oplus \mathbf{Y}^{\perp} .
$$

As immediate consequence of such orthogonal decompositions, the inf-sup conditions

$$
\inf _{\mathbf{u} \in \mathbf{Y}} \sup _{\boldsymbol{\tau} \in \mathbf{Q}} \frac{(\mathbf{u}, \operatorname{curl} \boldsymbol{\tau})}{\|\mathbf{u}\|_{\mathbf{R}}\|\boldsymbol{\tau}\|_{\mathbf{Q}}} \geq \frac{1}{\gamma} \quad \text { and } \quad \inf _{p \in W} \sup _{\mathbf{v} \in \mathbf{R}} \frac{(p, \operatorname{div} \mathbf{v})}{\|p\|_{W}\|\mathbf{v}\|_{\mathbf{R}}} \geq \frac{1}{\beta}
$$

hold for some constant $\gamma$ and $\beta$ which depends only on the domain $\Omega$. In fact, the spaces $\mathbf{X}^{\perp}$ and $\mathbf{Y}^{\perp}$ have the property that

$$
\left\|\boldsymbol{\tau}^{\perp}\right\|_{H(\text { curl })} \leq \gamma\left\|\operatorname{curl} \boldsymbol{\tau}^{\perp}\right\|_{L^{2}} \forall \boldsymbol{\tau}^{\perp} \in \mathbf{X}^{\perp} \text {, and }\left\|\mathbf{v}^{\perp}\right\|_{H(\operatorname{div})} \leq \beta\left\|\operatorname{div} \mathbf{v}^{\perp}\right\|_{L^{2}} \forall \mathbf{v}^{\perp} \in \mathbf{Y}^{\perp} .
$$

1.2. Well-posedness of the mixed variational formulation. Proceeding as in [20], with standard manipulation, we obtain the variational formulation of the mixed Brinkman problem (1.2).

Problem 1.1. Find $(\boldsymbol{\sigma}, \mathbf{u}, p) \in \mathbf{Q} \times \mathbf{R} \times W$ such that

$$
\left\{\begin{array}{llll}
m(\boldsymbol{\sigma}, \boldsymbol{\tau}) & -c^{*}(\mathbf{u}, \boldsymbol{\tau}) & =F(\boldsymbol{\tau}) & \forall \boldsymbol{\tau} \in \mathbf{Q} \\
-c(\boldsymbol{\sigma}, \mathbf{v}) & -a(\mathbf{u}, \mathbf{v})-d(\mathbf{u}, \mathbf{v}) \quad+b^{*}(p, \mathbf{v}) & =G(\mathbf{v}) & \forall \mathbf{v} \in \mathbf{R} \\
& b(\mathbf{u}, q) & =H(q) & \forall q \in Q
\end{array}\right.
$$


where

$$
\begin{array}{rc}
m(\boldsymbol{\sigma}, \boldsymbol{\tau})=(\boldsymbol{\sigma}, \boldsymbol{\tau}) & \boldsymbol{\sigma}, \boldsymbol{\tau} \in \mathbf{Q} \\
c(\boldsymbol{\sigma}, \mathbf{v})=\varepsilon(\operatorname{curl} \boldsymbol{\sigma}, \mathbf{v}) & \boldsymbol{\sigma} \in \mathbf{Q}, \mathbf{v} \in \mathbf{R}, \\
a(\mathbf{u}, \mathbf{v})=\varepsilon^{2}(\operatorname{div} \mathbf{u}, \operatorname{div} \mathbf{v}) & \mathbf{u}, \mathbf{v} \in \mathbf{R} \\
d(\mathbf{u}, \mathbf{v})=(k(\mathbf{x}) \mathbf{u}, \mathbf{v}) & \mathbf{u}, \mathbf{v} \in \mathbf{R}, \\
b(\mathbf{u}, q)=(\operatorname{div} \mathbf{u}, q) & \mathbf{u} \in \mathbf{R}, q \in Q
\end{array}
$$

$F \in \mathbf{Q}^{*}, G \in \mathbf{R}^{*}, H \in W^{*}$ are bounded functionals that take into account volume forces and boundary conditions.

The stability analysis of the mixed formulation was carried out by the authors in [20], following the analysis of the Hodge Laplacian in [6], [3]. Here, we summarize the main result.

Theorem 1.1. If $\varepsilon \geq 0$ and $k(x) \in L^{\infty}(\Omega) \cap L^{2}(\Omega), 0 \leq k_{\min } \leq k(\mathbf{x}) \leq k_{\max }$ almost everywhere in $\Omega$, then for given continuous linear functionals $F \in \mathbf{Q}^{*}, G \in \mathbf{R}^{*}, H \in W^{*}$ the generalized Brinkman problem (1.1) admits an unique solution and the following a priori estimate holds:

$$
\|\boldsymbol{\sigma}\|_{\mathbf{Q}}^{2}+\|\mathbf{u}\|_{\mathbf{R}}^{2}+\|p\|_{W}^{2} \leq C\left(\Omega, \varepsilon, k_{\min }, k_{\max }\right)\left(\|F\|_{\mathbf{Q}^{*}}^{2}+\|G\|_{\mathbf{R}^{*}}^{2}+\|H\|_{W}^{2}\right) .
$$

The main ingredients in the proof of the stability theorem are the inf-sup conditions (1.3) and the orthogonal decomposition of $\mathbf{Q}$ and $\mathbf{R}$. We refer to [20] for the details.

\section{Discretization}

Let us introduce the finite element spaces $\mathbf{Q}_{h} \subset \mathbf{Q}, \mathbf{R}_{h} \subset \mathbf{R}, W_{h} \subset W$. In order to achieve stability of the discretized problem, the discrete spaces $\mathbf{Q}_{h}, \mathbf{R}_{h}, W_{h}$ should preserve Hilbert structure of de Rham complex in the continuous case (see [6] for more details):

$$
\begin{aligned}
& \mathbf{Q} \rightarrow \text { curl } \rightarrow \mathbf{R} \rightarrow \operatorname{div} \rightarrow W \\
& \mathbf{Q}_{h} \rightarrow \operatorname{curl} \rightarrow \mathbf{R}_{h} \rightarrow \operatorname{div} \rightarrow W_{h} .
\end{aligned}
$$

A standard choice for numerical discretization of the Hodge Laplacian is the following. For a given integer $r \geq 0$, we let $\mathbf{Q}_{h}$ be the $(r+1)$-th order Nédélec finite elements ([16]), $\mathbf{R}_{h}$ the $r$-th order Raviart-Thomas finite elements ([18]), and $W_{h}$ the piecewise discontinuous polynomials finite element of degree $r$. A possible alternative, which also lead to a stable method, is to use continuous piecewise polynomials finite element of degree $r+1$ to discretize $\mathbf{Q}_{h}$ ([3]).

For such choice of discrete spaces, it is well-known (see e.g. [19]) that the inf-sup conditions (1.3) hold with $\beta$ and $\gamma$ independent from the mesh diameter $h$, and moreover

$$
\operatorname{curl} \mathbf{Q}_{h}=\mathbf{R}_{h} \text {, and } \operatorname{div} \mathbf{R}_{h}=W_{h} .
$$

In the following and in the numerical experiments, we will restrict ourselves to the case $r=0$, i.e. first order Nédélec elements, lowest order Raviart-Thomas elements, and piecewise constant elements. Such choice leads to linear decay of the discretization error, and it is optimal for in the case of low regularity in the analytical solution, due to discontinuity in the PDE coefficients.

The discrete Galerkin problem reads:

Problem 2.1. Find $\left(\boldsymbol{\sigma}_{h}, \mathbf{u}_{h}, p_{h}\right) \in \mathbf{Q}_{h} \times \mathbf{R}_{h} \times W_{h}$ such that

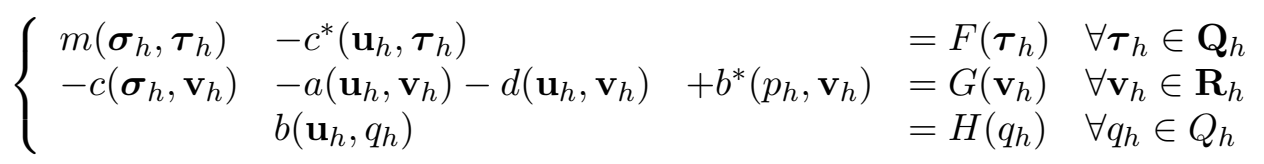


Let $\boldsymbol{\Sigma}, \mathbf{U}, P$ the finite element vectors collecting the degree of freedom $\boldsymbol{\sigma}_{h}^{i}, i=1, \ldots, \operatorname{dim}\left(\mathbf{Q}_{h}\right), \mathbf{u}_{h}^{i}$, $i=1, \ldots, \operatorname{dim}\left(\mathbf{R}_{h}\right)$ and $p_{h}^{i}, i=1, \ldots, \operatorname{dim}\left(W_{h}\right)$, and let $M, C, A, D, B$ the finite element matrices whose entries are given by

$$
\begin{aligned}
& M_{i, j}=m\left(\boldsymbol{\sigma}_{h}^{j}, \boldsymbol{\tau}_{h}^{i}\right)=\left(\boldsymbol{\sigma}_{h}^{j}, \boldsymbol{\tau}_{h}^{i}\right) \quad i, j=1, \ldots, \operatorname{dim}\left(\mathbf{Q}_{h}\right) \\
& C_{i, j}=c\left(\boldsymbol{\sigma}_{h}^{j}, \mathbf{v}_{h}^{i}\right)=\varepsilon\left(\operatorname{curl} \boldsymbol{\sigma}_{h}^{j}, \mathbf{v}_{h}^{i}\right) \quad i=1, \ldots, \operatorname{dim}\left(\mathbf{R}_{h}\right) j=1, \ldots, \operatorname{dim}\left(\mathbf{Q}_{h}\right) \\
& A_{i, j}=a\left(\mathbf{u}_{h}^{j}, \mathbf{v}_{h}^{i}\right)=\varepsilon^{2}\left(\operatorname{div} \mathbf{u}_{h}^{j}, \operatorname{div} \mathbf{v}_{h}^{i}\right) \quad i, j=1, \ldots, \operatorname{dim}\left(\mathbf{R}_{h}\right) \\
& D_{i, j}=d\left(\mathbf{u}_{h}^{j}, \mathbf{v}_{h}^{i}\right)=\left(k(\mathbf{x}) \mathbf{u}_{h}^{j}, \mathbf{v}_{h}^{i}\right) \quad i, j=1, \ldots, \operatorname{dim}\left(\mathbf{R}_{h}\right) \\
& B_{i, j}=b\left(\mathbf{u}_{h}^{j}, q_{h}^{i}\right)=\left(\operatorname{div} \mathbf{u}_{h}^{j}, q_{h}^{i}\right) \quad i=1, \ldots, \operatorname{dim}\left(W_{h}\right) j=1, \ldots, \operatorname{dim}\left(\mathbf{R}_{h}\right) .
\end{aligned}
$$

In algebraic form, we obtain the linear system

$$
\mathcal{B X}=\mathbf{B}
$$

where the block matrix $\mathcal{B}$ and block vectors $\mathbf{X}$ and $\mathbf{B}$ read:

$$
\mathcal{B}=\left[\begin{array}{ccc}
M & -C^{T} & 0 \\
-C & -A-D & B^{T} \\
0 & B & 0
\end{array}\right], \quad \mathbf{X}=\left[\begin{array}{c}
\Sigma \\
\mathbf{U} \\
P
\end{array}\right], \quad \mathbf{B}=\left[\begin{array}{c}
\mathbf{F} \\
\mathbf{G} \\
H
\end{array}\right] .
$$

We also introduce the augmented formulation of the Brinkman problem that will be used for the derivation of the preconditioner. Letting $M_{W}$ being the pressure mass matrix and $\gamma \in \mathbb{R}$ a positive number, the augmented matrix and right hand side have the form

$$
\mathcal{B}_{\gamma}=\left[\begin{array}{ccc}
M & -C^{T} & 0 \\
-C & -A-D-\gamma B^{T} M_{W}^{-1} B & B^{T} \\
0 & B & 0
\end{array}\right], \quad \mathbf{B}_{\gamma}=\left[\begin{array}{c}
\mathbf{F} \\
\mathbf{G}-\gamma B M_{W}^{-1} H \\
H
\end{array}\right] .
$$

\section{Preconditioning}

The discretized linear system (2.2) has the form of a symmetric saddle point problem, having $\operatorname{dim}\left(\mathbf{Q}_{h}\right)+\operatorname{dim}\left(W_{h}\right)$ positive eigenvalues and $\operatorname{dim}\left(\mathbf{R}_{h}\right)$ negative eigenvalues. An effective iterative methods to solve linear system with symmetric indefinite matrices is minres ([17]) with a symmetric positive definite preconditioner $\mathcal{P}$.

To derive the preconditioner, we follow the approach presented in $([15])$ to precondition symmetric saddle point problems in a Hilbert space setting. According to the authors, the mapping properties of the differential operators of the continuous problem suggest that block diagonal preconditioners are natural choices for saddle point problems. More specifically, given a stability estimate for the continuous problem in some functional spaces, the saddle point discrete system is spectrally equivalent to the block diagonal matrix, in which the blocks represent the discretization of the inner products in those spaces.

For some positive number $w_{Q}, w_{R}, w_{W}$, let us introduce the symmetric positive definite variational forms

$$
\begin{aligned}
& q\left(\boldsymbol{\sigma}_{h}, \boldsymbol{\tau}_{h}\right)=\left(\boldsymbol{\sigma}_{h}, \boldsymbol{\tau}_{h}\right)+w_{Q}\left(\operatorname{curl} \boldsymbol{\sigma}_{h}, \operatorname{curl} \boldsymbol{\tau}_{h}\right), \quad \boldsymbol{\sigma}_{h}, \boldsymbol{\tau}_{h} \in \mathbf{Q}_{h} \\
& r\left(\mathbf{u}_{h}, \mathbf{v}_{h}\right)=w_{R}\left(\mathbf{u}_{h}, \mathbf{v}_{h}\right)+w_{R}\left(\operatorname{div} \mathbf{u}_{h}, \operatorname{div} \mathbf{v}_{h}\right), \quad \mathbf{u}_{h}, \mathbf{v}_{h} \in \mathbf{R}_{h} \\
& w\left(p_{h}, q_{h}\right)=\quad w_{W}\left(p_{h}, q_{h}\right), \quad p_{h}, q_{h} \in W_{h} .
\end{aligned}
$$

The above forms define weighted inner products in $\mathbf{Q}, \mathbf{R}$ and $W$.

Therefore (based on [15]), an optimal preconditioner for the saddle point problem (2.2) is given by

$$
\mathcal{P}=\left[\begin{array}{ccc}
Q & 0 & 0 \\
0 & R & 0 \\
0 & 0 & W
\end{array}\right]
$$


where $Q, R, W$ are the matrix representation of the weighted inner products $q\left(\boldsymbol{\sigma}_{h}, \boldsymbol{\tau}_{h}\right), r\left(\mathbf{u}_{h}, \mathbf{v}_{h}\right)$, and $w\left(p_{h}, q_{h}\right)$.

In the constant coefficient case $k(\mathbf{x})=k_{0}$, the coercivity analysis of the Schur complement $D+A+$ $k_{0} B^{T} M_{W}^{-1} B+\varepsilon^{2} C M^{-1} C^{T}=k_{0} M_{\mathbf{R}}+\left(k_{0}+\varepsilon^{2}\right) B^{T} M_{W}^{-1} B+\varepsilon^{2} C M^{-1} C^{T}$ based on the uniform inf-sup condition provides the optimal weights

$$
w_{Q}=\frac{\varepsilon^{2}}{k_{0}+\varepsilon^{2}}, \quad w_{R}=k_{0}+\varepsilon^{2}, \quad w_{W}=\frac{1}{k_{0}+\varepsilon^{2}} .
$$

In the numerical results section, we verify mesh independence of this weighted norm preconditioner for constant coefficients. An approximated version of the preconditioner based on auxiliary space AMG for $H$ (curl) and $H(\mathrm{div})$ is also discussed. Finally, we extend such preconditioner to the case of non-constant coefficient $k(\mathbf{x})$, obtaining optimal convergence rates for smooth coefficients $k(\mathbf{x})$.

\section{Numerical Results}

The numerical results presented in this section are obtained using the finite element library MFEM [http://code.google.com/p/mfem/], developed at LLNL. MFEM is a general, modular, parallel C++ library for finite element methods research and development. It supports a wide variety of finite element spaces in 2D and 3D, as well as many bilinear and linear forms defined on them. It includes classes for dealing with various types of triangular, quadrilateral, tetrahedral and hexahedral meshes and their global and local refinement. Parallelization in MFEM is based on MPI, and it leads to high scalability in the finite element assembly procedure. It supports several solvers from the hypre library (http://www.llnl.gov/CASC/hypre/). In particular, in our work we used the auxiliary space algebraic multigrid solvers for $H$ (curl) and $H$ (div) ([12], [13]).

The initial meshes used in our simulation were generated with the unstructured mesh generator netgen [http://www.hpfem.jku.at/netgen/].

The numerical results presented in this section were obtained on hera, a high performance computer at LLNL. Hera has a total of 864 nodes connected by InfiniBand DDR (Mellanox). Each node has 16 AMD Quad-Core Opteron 2.3Ghz cpus, and 32GB of memory. Hera is running CHAOS 4.4, a linux kernel developed at LLNL, specific for high performance computing.

Our code was compiled with the Intel mpiicc and mpiicpc compilers version 11.1.046.

4.1. Constant coefficient weak scalability test. We study the performance of the proposed preconditioner in the case of constant coefficients. In particular, we present results relative to the augmented formulation of the Brinkman problem and the block diagonal preconditioner $\mathcal{P}$, where the weights $w_{Q}$, $w_{R}, w_{W}$ are chosen accordingly to (3.3).

Two cases are analyzed:

- exact preconditioner: the blocks $P_{Q}^{-1}, P_{R}^{-1}$ are solved exactly by using the preconditioned conjugate gradient method.

- AMG preconditioner: the blocks $\hat{P}_{Q}^{-1}, \hat{P}_{R}^{-1}$ are given by one V-cycle for the auxiliary space AMG for $H$ (curl) and $H$ (div) problem respectively.

In practice the AMG version of the preconditioner out-performs the exact one, but we remark the theoretical importance of the latter, since it allows us to confirm the theoretical mesh independence of the preconditioner.

For this test we used three different meshes in which the number of elements doubles from the previous to the next. By cycling the three meshes and by using uniform refinements on each of them, we are able to build a sequence of Brinkman problem whose size doubles each time. The sizes of the three meshes at the coarser level of refinement are given in Table 1. We use Metis ([11]) for the partitioning of the mesh in aggregates. 


\begin{tabular}{|c|c|c|c|}
\hline & $n_{t}$ & $n_{f}$ & $n_{e}$ \\
\hline mesh 1 & 30336 & 62336 & 37940 \\
mesh 2 & 57472 & 118304 & 72164 \\
mesh 3 & 129920 & 266448 & 161602 \\
\hline
\end{tabular}

TABLE 1. Number of elements $n_{t}$, faces $n_{f}$, and edges $n_{e}$ on the coarser level of each unstructured mesh.

Through the simulations we chose the number of processes in order to keep the number of unknowns per processor as constant as possible as we increased the total number of unknowns. We equally distribute the number of processes on each node of the parallel machine and we try to balance concurrency inside the node, on the one hand, with communications between nodes, on the other.

In Table 2 we verify the mesh independence of the exact version of the preconditioner. The outer tolerance of MINRES was set to $10^{-10}$ while the inner tolerance of PCG was set to $10^{-12}$. The number of iterations is uniformly bounded for every value of $k$. Beside the case $k=10^{6}$ in which we observe a moderate increase of the iteration numbers, the preconditioner shows a perfectly mesh independence behavior.

In Table 3 we show the number of iterations when using a single V-cycle of the auxiliary space AMG preconditioners for $H$ (curl) and $H$ (div), keeping all other parameters in the test the same as before. For fixed $k$, we observe a moderate increase of the number of iterations as the number of unknowns is growing. This is expected, given the particular choice of the parameters in the V-cycle which are made in order to minimize wall time instead of number iterations. We refer to [12] for a more detailed discussion about the choice of the multigrid parameters and their effects on number of iterations for the auxiliary space AMG preconditioner for $H$ (curl) problems. Regarding the dependency of the iteration count with respect to the value of $k$ we notice that in the AMG version the number of iterations (even if higher) is quite homogeneous with respect to $k$ (for fixed mesh size).

Finally, in Table 4 we report the wall time to set-up the preconditioner $\left(t_{\text {setup }}\right)$ and to solve iteratively the linear system with minres $\left(t_{\text {solve }}\right)$. Timings are computed by using the mpi function MPI_Wtime () . The computation of the preconditioner consists in two phases. First we assemble the finite element matrices for the variational forms $q\left(\boldsymbol{\sigma}_{h}, \boldsymbol{\tau}_{h}\right), r\left(\mathbf{u}_{h}, \mathbf{v}_{h}\right)$, and $w\left(p_{h}, q_{h}\right)$. Then we compute the auxiliary space interpolators, restriction matrices, and coarse matrices and solvers needed for to apply the Vcycle. We show only one column for $t_{\text {setup }}$ since the preconditioner setup is independent from the values of $k$. $t_{\text {setup }}$ is usually negligible compared to $t_{\text {solve }}$ (less than $10 \%$ in all cases), and it scales well (even if not perfectly) with the number of processes. The fact that for $n p=16$ and $n p=128$ it is faster than in the cases $n p=8$ and $n p=64$, respectively, may suggest some load unbalance due to the partition of the meshes in the latter case. With respect to the solution times $t_{\text {solve }}$ we notice that for a fixed problem size they tend to decrease as we approach the Darcy limit since less iterations are required to converge. For fixed $k$ the scaling of $t_{\text {solve }}$ with respect to the number of processors is similar to the one reported in [12] up to 128 processes, but we observe a severe loss of scalability when we use 256 processes. Possible causes of this loss of performance could be not perfect load balancing and hardware configuration issues, which are beyond the scope of this work.

4.2. The case of non-constant coefficients. Now we consider the case of non-constant coefficient $k(\mathbf{x})$. Being $\Omega=[0,1]^{3}$ and $c \leq 1$ a positive number, we consider the case

$$
k(\mathbf{x})=\frac{1}{\sin (\pi y) \sin (\pi z)+c} \quad \forall(x, y, z) \in \Omega .
$$

The number $c$ controls how large are the variations in the coefficient $k(\mathbf{x})$, since $k(\mathbf{x})$ ranges between $k_{\min } \sim 1$ and $k_{\max } \sim \frac{1}{c}$. We let the viscosity $\nu=\varepsilon^{2}=1$ and we choose the right hand side and natural 
PANAYOT S. VASSILEVSKI AND UMBERTO VILLA

\begin{tabular}{|c|c|c|c|c|c|c|c|}
\hline $\mathrm{N}$ & \multicolumn{6}{|c|}{ Number of MINRES iterations (Exact Preconditioner) } \\
\hline & $k=0$ & $k=10^{-6}$ & $k=10^{-3}$ & $k=1$ & $k=10^{3}$ & $k=10^{6}$ & $\nu=0$ \\
\hline 130612 & 16 & 16 & 16 & 18 & 30 & 17 & 10 \\
247940 & 16 & 16 & 16 & 18 & 30 & 18 & 10 \\
557970 & 16 & 16 & 16 & 18 & 30 & 20 & 10 \\
\hline 1027944 & 16 & 16 & 16 & 18 & 30 & 20 & 10 \\
1949480 & 16 & 16 & 16 & 18 & 30 & 22 & 10 \\
4396980 & 16 & 16 & 16 & 18 & 30 & 23 & 10 \\
\hline 8156368 & 16 & 16 & 16 & 18 & 30 & 23 & 10 \\
15460560 & 16 & 16 & 16 & 18 & 30 & 25 & 10 \\
34910120 & 16 & 16 & 16 & 18 & 30 & 27 & 10 \\
\hline
\end{tabular}

TABLE 2. Number of MINRES iterations with the exact preconditioner for different values of $k . N$ represents the total number of unknowns.

\begin{tabular}{|c|c|c|c|c|c|c|c|}
\hline & \multicolumn{6}{|c|}{ Number of MINRES iterations (AMG Preconditioner) } \\
\hline $\mathrm{N}$ & $k=0$ & $k=10^{-6}$ & $k=10^{-3}$ & $k=1$ & $k=10^{3}$ & $k=10^{6}$ & $\nu=0$ \\
\hline 130612 & 44 & 44 & 44 & 36 & 37 & 32 & 21 \\
247940 & 48 & 48 & 48 & 40 & 39 & 34 & 23 \\
557970 & 51 & 51 & 51 & 46 & 43 & 37 & 24 \\
\hline 1027944 & 57 & 57 & 57 & 48 & 49 & 39 & 26 \\
1949480 & 60 & 60 & 60 & 51 & 50 & 40 & 27 \\
4396980 & 61 & 61 & 61 & 52 & 52 & 42 & 28 \\
\hline 8156368 & 68 & 69 & 68 & 61 & 55 & 43 & 28 \\
15460560 & 72 & 73 & 72 & 64 & 58 & 44 & 30 \\
34910120 & 72 & 72 & 72 & 65 & 59 & 45 & 30 \\
\hline
\end{tabular}

TABLE 3. Number of MINRES iterations with the AMG preconditioner for different values of $k . N$ represents the total number of unknowns.

\begin{tabular}{|c|c|c|c|c|c|c|c|c|c|c|}
\hline & & & \multicolumn{7}{|c|}{$t_{\text {solve }}$ (AMG Preconditioner) } & $t_{\text {setup }}$ \\
\hline$n n$ & $n p$ & $\mathrm{~N}$ & $k=0$ & $k=10^{-6}$ & $k=10^{-3}$ & $k=1$ & $k=10^{3}$ & $k=10^{6}$ & $\nu=0$ & \\
\hline 1 & 1 & 130612 & 15.2 & 15.1 & 15.1 & 12.7 & 13.0 & 11.3 & 8.0 & 0.71 \\
1 & 2 & 247940 & 17.7 & 17.7 & 17.7 & 15.1 & 14.7 & 13.0 & 9.5 & 0.96 \\
1 & 4 & 557970 & 22.6 & 22.5 & 22.5 & 19.5 & 19.4 & 16.8 & 11.9 & 1.28 \\
\hline 8 & 8 & 1027944 & 25.6 & 25.2 & 24.9 & 21.6 & 21.9 & 17.5 & 13.0 & 1.47 \\
8 & 16 & 1949480 & 26.8 & 26.9 & 26.8 & 22.8 & 22.6 & 18.4 & 13.7 & 1.42 \\
8 & 32 & 4396980 & 33.0 & 33.0 & 33.1 & 28.3 & 28.6 & 22.9 & 17.0 & 1.74 \\
\hline 64 & 64 & 8156368 & 36.2 & 36.8 & 36.7 & 33.2 & 30.6 & 24.5 & 20.1 & 2.15 \\
64 & 128 & 15460560 & 45.3 & 44.8 & 44.9 & 41.5 & 35.7 & 28.3 & 22.0 & 1.76 \\
64 & 256 & 34910120 & 90.0 & 91.7 & 91.0 & 83.2 & 76.7 & 52.3 & 43.6 & 2.56 \\
\hline
\end{tabular}

TABLE 4. Computational cost of the AMG preconditioner. $n n$ is the number of nodes used, $n p$ is the number of processes, $N$ the total number of degree of freedom, $t_{\text {solve }}$ and $t_{\text {setup }}$ measures the time in seconds to solve the linear system and to assemble the preconditioner, respectively.

boundary conditions on $\partial \Omega$ such that the analytical solution is given by

$$
\boldsymbol{\sigma}_{\text {exact }}=\left[\begin{array}{c}
0 \\
\pi \sin (\pi y) \cos (\pi z) \\
-\pi \cos (\pi y) \sin (\pi x)
\end{array}\right] \quad \mathbf{u}_{\text {exact }}=\left[\begin{array}{c}
\sin (\pi y) \sin (\pi z) \\
0 \\
0
\end{array}\right] \quad p_{\text {exact }}=-x .
$$




\begin{tabular}{|c|c|c|c||c|c|c|}
\hline & \multicolumn{3}{|c|}{ Exact $n_{\text {it }}$} & \multicolumn{3}{c|}{ AMG $n_{\text {it }}$} \\
\hline$n_{\text {ref }}$ & $\frac{1}{c}=1$ & $\frac{1}{c}=10^{3}$ & $\frac{1}{c}=10^{6}$ & $\frac{1}{c}=1$ & $\frac{1}{c}=10^{3}$ & $\frac{1}{c}=10^{6}$ \\
\hline 0 & 19 & 30 & 30 & 26 & 35 & 36 \\
1 & 19 & 29 & 30 & 32 & 37 & 38 \\
2 & 19 & 29 & 32 & 46 & 45 & 48 \\
3 & 19 & 27 & 32 & 52 & 49 & 53 \\
4 & 19 & 27 & 32 & 63 & 61 & 61 \\
5 & - & - & - & 74 & 70 & 70 \\
\hline
\end{tabular}

TABLE 5. Performances of the exact and AMG preconditioner for variable coefficient problem. The quantity $\frac{1}{c} \sim \frac{k_{\max }}{k_{\min }}$ determines how large it is the variation in the PDE coefficient $k(\mathbf{x}), n_{\text {ref }}$ represents the number of uniform mesh refinements, $n_{\text {it }}$ the number of preconditioned minres iterations to achieve a relative reduction of the residual norm up to $10^{-10}$.

The computational domain $\Omega$ is discretized with an initial unstructured tetrahedral mesh with 474 elements. The original mesh is then uniformly refined 5 times, where each element of the mesh is divided in 8 using bisection. The total number of degree of freedoms ranges from around 2 thousand dofs on the coarsest mesh up to 65 millions on the finest mesh.

For this test, we extend the augmentation technique discussed before to the case of non constant coefficient. In particular, we solve the augmented saddle point problem

$$
\left\{\begin{array}{c}
\left(\boldsymbol{\sigma}_{h}, \boldsymbol{\tau}_{h}\right)-\varepsilon\left(\mathbf{u}_{h}, \operatorname{curl} \boldsymbol{\tau}_{h}\right)=F\left(\boldsymbol{\tau}_{h}\right) \quad \forall \boldsymbol{\tau}_{h} \in \mathbf{Q}_{h} \\
-\varepsilon\left(\operatorname{curl} \boldsymbol{\sigma}_{h}, \mathbf{v}_{h}\right)-\left(k(\mathbf{x}) \mathbf{u}_{h}, \mathbf{v}_{h}\right)-\left(\left(k(\mathbf{x})+\varepsilon^{2}\right) \operatorname{div} \mathbf{u}_{h}, \operatorname{div} \mathbf{v}_{h}\right)+\left(p_{h}, \operatorname{div} \mathbf{u}_{h}\right)= \\
G\left(\mathbf{v}_{h}\right)+H\left(k(\mathbf{x}) \operatorname{div} \mathbf{v}_{h}\right) \quad \forall \mathbf{v}_{h} \in \mathbf{R}_{h} \\
\left(\operatorname{div} \mathbf{u}_{h}, q_{h}\right)=H\left(q_{h}\right) \quad \forall q_{h} \in W_{h}
\end{array}\right.
$$

preconditioned by a block-diagonal preconditioner with blocks corresponding to the following bilinear forms:

$$
\begin{cases}\left(\boldsymbol{\sigma}_{h}, \boldsymbol{\tau}_{h}\right)+\varepsilon^{2}\left(\frac{1}{k(\mathbf{x})+\varepsilon^{2}} \operatorname{curl} \boldsymbol{\sigma}_{h}, \operatorname{curl} \boldsymbol{\tau}_{h}\right) & \boldsymbol{\sigma}_{h}, \boldsymbol{\tau}_{h} \in \mathbf{Q}_{h} \\ \left(\left(k(\mathbf{x})+\varepsilon^{2}\right) \mathbf{u}_{h}, \mathbf{v}_{h}\right)+\left(\left(k(\mathbf{x})+\varepsilon^{2}\right) \operatorname{div} \mathbf{u}_{h}, \operatorname{div} \mathbf{v}_{h}\right) & \mathbf{u}_{h}, \mathbf{v}_{h} \in \mathbf{R}_{h} \\ \left(\frac{1}{k(\mathbf{x})+\varepsilon^{2}} p_{h}, q_{h}\right) & p_{h}, q_{h} \in W_{h} .\end{cases}
$$

In Table 5, we report the number of minres iterations for the solutions of the Brinkman problem with variable coefficients (stopping criterion: norm of the relative residual less or equal to $10^{-10}$ ). We show both the exact and inexact block-diagonal preconditioner. The inexact preconditioner consists of one V-cycle of the auxiliary space AMGs applied to the weighted $H$ (curl) and $H$ (div) variational forms in (4.3). The qualitatively behavior of the preconditioner is similar to the case of constant coefficients in Table 3.

\section{Conclusion}

In this paper we constructed an efficient and scalable preconditioner for the mixed formulation of the Brinkman problem proposed by the authors in [20]. The algebraic saddle point system obtained after finite elements discretization can be efficiently solved with Krylov iterative methods and a block diagonal AMG preconditioner. In particular, we used the auxiliary space algebraic multigrid preconditioners for $H$ (curl) and $H$ (div) for the vorticity and velocity block respectively, and diagonal scaling for the pressure block. In the case of constant or smooth PDE coefficients, the proposed preconditioner 
exhibits fairly scalable properties and it is robust with respect to a wide range of values of the inverse permeability coefficient $k(x)$.

\section{REFERENCES}

[1] Grgoire Allaire. Homogenization of the Navier-Stokes equations in open sets perforated with tiny holes I. Abstract framework, a volume distribution of holes. Archive for Rational Mechanics and Analysis, 113:209-259, 1991. 10.1007/BF00375065.

[2] Grgoire Allaire. Homogenization of the Navier-Stokes equations in open sets perforated with tiny holes II: Non-critical sizes of the holes for a volume distribution and a surface distribution of holes. Archive for Rational Mechanics and Analysis, 113:261-298, 1991. 10.1007/BF00375066.

[3] Douglas N Arnold, Richard S Falk, and Jay Gopalakrishnan. Mixed finite element approximation of the vector Laplacian with Dirichlet boundary conditions. arXiv 1109.3668, submitted to Mathematical Models \& Methods in Applied Sciences, 2011.

[4] Douglas N Arnold, Richard S. Falk, and Ragnar Winter. Preconditioning in H(div) and applications. Mathematics of Computations, 66:957 - 984, 1997

[5] Douglas N Arnold, Richard S. Falk, and Ragnar Winter. Multigrid in H(div) and H(curl). Numerische Mathematik, $85: 197-217,2000$

[6] Douglas N. Arnold, Richard S. Falk, and Ragnar Winther. Finite element exterior calculus: from Hodge theory to numerical stability. Bull. Amer. Math. Soc. (N.S.), 47:281-354, 2010. DOI: 10.1090/S0273-0979-10-01278-4.

[7] Erik Burman and Peter Hansbo. Stabilized Crouzeix-Raviart element for the Darcy-Stokes problem. Numerical Methods for Partial Differential Equations, 21(5):986-997, 2005.

[8] Erik Burman and Peter Hansbo. A unified stabilized method for Stokes' and Darcy's equations. Journal of Computational and Applied Mathematics, 198(1):35 - 51, 2007.

[9] M.R. Correa and A.F.D. Loula. A unified mixed formulation naturally coupling Stokes and Darcy flows. Computer Methods in Applied Mechanics and Engineering, 198(33-36):2710 - 2722, 2009.

[10] hypre: High performance preconditioners. http://www.llnl.gov/CASC/hypre/.

[11] George Karypis and Vipin Kumar. MeTis: Unstructured Graph Partitioning and Sparse Matrix Ordering System, Version 4.0. http://www.cs.umn.edu/ metis, 2009.

[12] Tzanio Kolev and Panayot S. Vassilevski. Parallel Auxiliary Space AMG for H(curl) Problems. J. of Computational Mathematics, 27, 2009.

[13] Tzanio Kolev and Panayot S. Vassilevski. Parallel Auxiliary Space AMG Solver for H(div) Problems. Technical Report LLNL-JRNL-520391, December 15, 2011.

[14] Kent Andre Mardal, Xue-Cheng Tai, and Ragnar Winther. A Robust Finite Element Method for Darcy-Stokes Flow. SIAM J. Numer. Anal., 40:1605-1631, May 2002.

[15] Kent-Andre Mardal and Ragnar Winther. Preconditioning discretizations of systems of partial differential equations. Numerical Linear Algebra with Applications, 18(1):1-40, 2011.

[16] J. C. Nedelec. Mixed finite elements in $R^{3}$. Numerische Mathematik, 35:315-341, 1980. 10.1007/BF01396415.

[17] C. C. Paige and M. A. Saunders. Solution of sparse indefinite systems of linear equations. SIAM J. Numerical Analysis, pages 617-629, 1975 .

[18] P. A. Raviart and J. M. Thomas. A mixed finite element method for 2nd order elliptic problems. Mathematical Aspects of the Finite Element Method, Lecture Notes in Mathematics, 606:292-315, 1977.

[19] Panayot S. Vassilevski. Multilevel Block Factorization Preconditioners. Springer, New York, 2008.

[20] Panayot S. Vassilevski and Umberto Villa. A mixed formulation for the Brinkman problem. in preparation, 2012.

[21] X.P. Xie, J.C. Xu, and G.R Xue. Uniformly-stable finite element methods for Darcy-Stokes-Brinkman models . J. Comput. Math., 2008

Center for Applied Scientific Computing, Lawrence Livermore National Laboratory, P.O. Box 808, L-561, Livermore, CA 94551, U.S.A.

E-mail address: panayot@llnl.gov

Department of Mathematics and Computer Science, Emory University, Atlanta, Ga

E-mail address: uvilla@emory.edu 